\title{
PAPR MINIMIZATION THROUGH SPATIO-TEMPORAL SYMBOL-LEVEL PRECODING FOR THE NON-LINEAR MULTI-USER MISO CHANNEL
}

\author{
Danilo Spano, Maha Alodeh, Symeon Chatzinotas, and Björn Ottersten \\ Interdisciplinary Centre for Security Reliability and Trust (SnT), University of Luxembourg \\ \{danilo.spano, maha.alodeh, symeon.chatzinotas, and bjorn.ottersten\}@uni.lu
}

\begin{abstract}
Symbol-level precoding (SLP) is a promising technique which allows to constructively exploit the multi-user interference in the downlink of multiple antenna systems. Recently, this approach has also been used in the context of non-linear systems for reducing the instantaneous power imbalances among the antennas. However, previous works have not exploited SLP to improve the dynamic properties of the waveforms in the temporal dimension, which are fundamental for non-linear systems. To fill this gap, this paper proposes a novel precoding method, referred to as spatio-temporal SLP, which minimizes the peak-to-average power ratio of the transmitted waveforms both in the spatial and in the temporal dimensions, while at the same time exploiting the constructive interference effect. $\mathrm{Nu}-$ merical results are presented to highlight the enhanced performance of the proposed scheme with respect to state of the art SLP techniques, in terms of power distribution and symbol error rate over non-linear channels.
\end{abstract}

Index Terms - Symbol-level precoding, Multi-user MISO, PAPR, Non-linear channel.

\section{INTRODUCTION}

The utilization of multi-antenna transmitters relying on full frequency reuse has revealed to be an effective strategy towards fulfilling the constantly increasing throughput requirements of current wireless communication systems. As a consequence, in recent years precoding has been a prolific research area, due to its ability to handle the interference arising between simultaneous transmissions addressed to different co-channel users.

The conventional precoding strategies aim at mitigating the interference by exploiting the knowledge of the channel state information (CSI), through the design a precoding weight matrix (or precoder) to be applied to the multiple data streams [1-3]. On the other hand, symbol-level precoding (SLP) is a novel strategy [4-13] which designs the transmitted signals on a symbol-by-symbol basis, using the knowledge of both the CSI and the data information. The objective of SLP is not to eliminate the interference, but rather to control it so as to achieve a constructive interference effect at each receiver. Different optimization strategies have been considered in the literature for SLP. In [6] the sum power minimization and the max-min fair problem were solved for PSK modulations. Extensions of such works include optimization strategies for multi-level modulations [7]

This work is supported by H2020 project SANSA (Shared Access Terrestrial-Satellite Backhaul Network enabled by Smart Antennas), FNR projects PROSAT (on-board PROcessing techniques for high throughput SATellites ) and SATSENT (SATellite SEnsor NeTworks for spectrum monitoring), FNR-EPSRC project CI-PHY (Exploiting interference for physical layer security in 5G networks), and FNR-AFR project BroadSat. and more flexible approaches for exploiting the constructive interference [8]. A more detailed review of SLP can be found in [14].

SLP schemes have been recently proposed by the authors also in the context of non-linear systems [10-13], where the per-antenna high-power amplifiers (HPAs) [15] introduce an amplitude and phase distortion on the transmitted streams. The resulting performance degradation depends on the power variation of the signals both in the temporal and in the spatial dimensions. The latter one is referred to the variation of the instantaneous transmit power among the different antennas, which causes a differential distortion effect (the differential phase shift discussed in $[12,13])$ and therefore deteriorates the performance. The schemes of [10-13] tackle this problem by reducing the instantaneous power imbalances among the antennas, for each symbol slot. Nonetheless, these SLP schemes fail at improving the power dynamic of the waveforms in the temporal dimension, which is fundamental with respect to non-linearities, since they work on a symbol-by-symbol basis. In order to fill this gap, this work proposes a novel SLP method, referred to as spatio-temporal SLP, which is able to optimize the power dynamic of the waveforms both in the spatial and in the temporal dimensions, thus further improving the robustness of the signals to non-linear effects. Specifically, the proposed scheme performs a minimization of the spatio-temporal peak-to-average power ratio (PAPR) of the transmitted waveforms, under Quality-of-Service (QoS) constraints, for multi-level modulation schemes ${ }^{1}$. It should be mentioned that a first spatio-temporal SLP formulation has been presented by the authors in [17], which however is not addressed to non-linear systems and therefore follows a different optimization strategy.

Notation: $(\cdot)^{T}$ denotes matrix transpose, while $(\cdot)^{\dagger}$ denotes matrix conjugate transpose. $|\cdot|$ and $\angle(\cdot)$ denote the amplitude and the phase, respectively, while $\operatorname{Re}(\cdot)$ and $\operatorname{Im}(\cdot)$ are the real and imaginary parts, and $\iota$ is used to denote the imaginary unit. Moreover, $\|\cdot\|$ and $\|\cdot\|_{\infty}$ represent the Euclidean norm and the $1_{\infty}$ norm , respectively. $\operatorname{Pr}(\cdot)$ denotes the probability of an event, while $\mathbb{E}(\cdot)$ denotes the statistical expectation. Finally, $\boldsymbol{I}_{a}$ denotes the identity matrix of size $a \times a$, while $\otimes$ denotes the Kronecker product.

\section{SYSTEM MODEL}

Let us consider a single-cell multiple-antenna downlink scenario, where a base-station delivers $K$ independent data streams to $K$ single-antenna user terminals through $N$ transmit antennas, with $N \geq K$. Each data stream is divided in blocks of $S$ symbols,

\footnotetext{
${ }^{1}$ There are a number of works, such as [16], proposing precoding techniques for PAPR reduction in massive MIMO systems. Nevertheless, the scheme herein proposed differs from them as it exploits the constructive interference effect of SLP previously discussed. Further, it does not rely on massive MIMO architectures.
} 


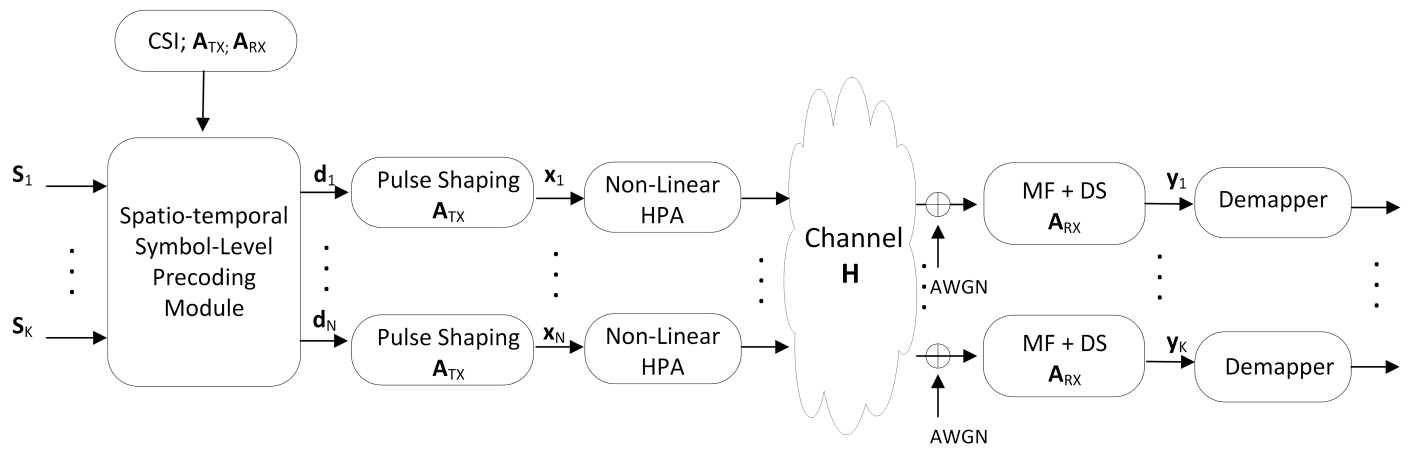

Fig. 1: Block scheme of the considered system model relying on spatio-temporal symbol-level precoding.

and the channel is assumed to be quasi-static flat fading. Considering a data block, we can define the data information matrix $\boldsymbol{S}=\left[\boldsymbol{s}_{1}^{T} \ldots \boldsymbol{s}_{K}^{T}\right]^{T} \in \mathbb{C}^{K \times S}$, which aggregates the symbol streams to be delivered to the different users. Similarly, we aggregate in the matrix $\boldsymbol{D}=\left[\boldsymbol{d}_{1}^{T} \ldots \boldsymbol{d}_{N}^{T}\right]^{T} \in \mathbb{C}^{N \times S}$ the precoded symbol streams feeding the transmit filters. In fact, each symbol stream has to undergo pulse shaping before the actual transmission. The pulse shaping operation is performed using a unit energy symmetric pulse waveform $\alpha(t)$. Denoting by $T$ the symbol period and by $\xi$ the oversampling factor, the transmitted waveform for the generic $n$-th antenna can be represented through its discrete samples spaced by $t_{s}=\frac{T}{\xi}$, as follows:

$$
x_{n}[l]=\sum_{i=1}^{S} d_{n}[i] \alpha\left[(l-1) t_{s}-(i-1) T\right], \quad l=1, \ldots, \xi S,
$$

where $d_{n}[i]$ is the $i$-th element of the symbol vector $\boldsymbol{d}_{n}$, which in turn is the $n$-th row of $\boldsymbol{D}$. By aggregating the output (oversampled) signals from all the antennas in a matrix $\boldsymbol{X}=\left[\boldsymbol{x}_{1}^{T} \ldots \boldsymbol{x}_{N}^{T}\right]^{T} \in$ $\mathbb{C}^{N \times \xi S}$, the pulse shaping operation can be represented in a compact matrix form as $\boldsymbol{X}=\boldsymbol{D} \boldsymbol{A}_{\mathrm{TX}}$, where $\boldsymbol{A}_{\mathrm{TX}} \in \mathbb{R}^{S \times \xi S}$ is a block Toeplitz matrix having as $(i, l)$-th element:

$$
\left[\boldsymbol{A}_{\mathrm{TX}}\right]_{(i, l)}=\alpha\left[(l-1) t_{s}-(i-1) T\right] .
$$

According to the well-known multi-user MISO channel model, the received symbols at the users can be written in matrix form as:

$$
\tilde{\boldsymbol{Y}}=\boldsymbol{H} \boldsymbol{X}+\tilde{\boldsymbol{Z}},
$$

where the matrix $\tilde{\boldsymbol{Y}}=\left[\tilde{\boldsymbol{y}}_{1}^{T} \ldots \tilde{\boldsymbol{y}}_{K}^{T}\right]^{T} \in \mathbb{C}^{K \times n_{s} S}$ represents the received samples at the $K$ users, $\boldsymbol{H}=\left[\boldsymbol{h}_{1}^{T} \ldots \boldsymbol{h}_{K}^{T}\right]^{T} \in \mathbb{C}^{K \times N}$ is the channel matrix modeling the interference among the different data streams, and $\tilde{\boldsymbol{Z}}=\left[\tilde{\boldsymbol{z}}_{1}^{T} \ldots \tilde{\boldsymbol{z}}_{K}^{T}\right]^{T} \in \mathbb{C}^{K \times \xi S}$ models the Additive White Gaussian Noise (AWGN). In order to obtain the received signals at the users in the symbol domain, the matched filtering and downsampling operation needs to be modeled. This can be done again in a matrix form, using the block Toeplitz matrix $\boldsymbol{A}_{\mathrm{RX}} \in \mathbb{R}^{\xi S \times S}$, which can be defined in the same fashion of (2). Overall, grouping the received symbols at the $K$ users in a matrix $\boldsymbol{Y}=\left[\boldsymbol{y}_{1}^{T} \ldots \boldsymbol{y}_{K}^{T}\right]^{T} \in \mathbb{C}^{K \times S}$, we can write the global communication model as:

$$
\boldsymbol{Y}=\tilde{\boldsymbol{Y}} \boldsymbol{A}_{\mathrm{RX}}=\boldsymbol{H} \boldsymbol{X} \boldsymbol{A}_{\mathrm{RX}}+\tilde{\boldsymbol{Z}} \boldsymbol{A}_{\mathrm{RX}}=\boldsymbol{H} \boldsymbol{D} \boldsymbol{A}+\boldsymbol{Z},
$$

where $\boldsymbol{A}=\boldsymbol{A}_{\mathrm{TX}} \boldsymbol{A}_{\mathrm{RX}} \in \mathbb{R}^{S \times S}$ represents the combination of the filters at the transmitter and at the receiver, while $\boldsymbol{Z}=\tilde{\boldsymbol{Z}} \boldsymbol{A}_{\mathrm{RX}} \in$ $\mathbb{C}^{K \times S}$ is the noise in the symbol domain. Without loss of generality, the noise power is assumed to be 1 .

As anticipated, it should be taken into account how the system model introduced in (3) is actually degraded by the non-linear effects introduced by the per-antenna HPAs. A practical example of non-linear HPA is given in Fig. 2, where the (normalized) AM-AM and AM-PM characteristics of the non-linearized traveling-wavetube amplifier (TWTA) model of [18] are represented ${ }^{2}$. Such characteristics clearly show the introduced amplitude and phase distortion. The complete system model is represented in the block scheme of Fig. 1, where it is clear how the symbol matrix $\boldsymbol{D}$ is obtained as output of a spatio-temporal precoding module, which takes as input the CSI, i.e. an estimate of $\boldsymbol{H}$, the filters matrices $\boldsymbol{A}_{\mathrm{TX}}$ and $\boldsymbol{A}_{\mathrm{RX}}$ and the data information matrix $\boldsymbol{S}$. Differently than in previous SLP works $[10,12]$, the model in (3) represents the signals not only in the spatial dimension (i.e., how they vary between the antennas), but also in the temporal dimension, considering a whole block of $S$ symbols per stream and the oversampled transmitted waveforms through $\boldsymbol{X}$. This feature allows to take an important step forward in SLP towards counteracting the non-linear effects. In fact, with the introduced model it is possible to design the matrix $\boldsymbol{D}$, namely the precoded symbol streams feeding the transmit filters, by optimizing the power dynamic of the transmitted samples $\boldsymbol{X}$ both in the spatial and in the temporal dimensions, thus making the signals more robust to non-linear effects.

In order to facilitate the formulation of the proposed optimization scheme, discussed in the next section, it is convenient to further manipulate the model of (3) by vectorizing the introduced signal matrices over the temporal dimension (rows first). Hence, we model the data information streams through the vector $\boldsymbol{s}=\operatorname{vec}\left(\boldsymbol{S}^{T}\right)=$ $\left[\boldsymbol{s}_{1} \ldots \boldsymbol{s}_{K}\right]^{T} \in \mathbb{C}^{K S \times 1}$, the designed symbol streams through $\boldsymbol{d}=\operatorname{vec}\left(\boldsymbol{D}^{T}\right)=\left[\boldsymbol{d}_{1} \ldots \boldsymbol{d}_{K}\right]^{T} \in \mathbb{C}^{N S \times 1}$, the transmitted signals through $\boldsymbol{x}=\operatorname{vec}\left(\boldsymbol{X}^{T}\right)=\left[\boldsymbol{x}_{1} \ldots \boldsymbol{x}_{N}\right]^{T} \in \mathbb{C}^{N \xi S \times 1}$, the noise through $\boldsymbol{z}=\operatorname{vec}\left(\boldsymbol{Z}^{T}\right)=\left[\boldsymbol{z}_{1} \ldots \boldsymbol{z}_{K}\right]^{T} \in \mathbb{C}^{K S \times 1}$, and the received symbols through $\boldsymbol{y}=\operatorname{vec}\left(\boldsymbol{Y}^{T}\right)=\left[\boldsymbol{y}_{1} \ldots \boldsymbol{y}_{K}\right]^{T} \in \mathbb{C}^{K S \times 1}$. Accordingly, the relation between $\boldsymbol{d}$ and $\boldsymbol{x}$ can be written as $\boldsymbol{x}=\left(\boldsymbol{I}_{N} \otimes \boldsymbol{A}_{\mathrm{TX}}^{T}\right) \boldsymbol{d}$, and the communication model can be formalized as:

$$
\boldsymbol{y}=\left(\boldsymbol{H} \otimes \boldsymbol{A}^{T}\right) \boldsymbol{d}+\boldsymbol{z}=\boldsymbol{G} \boldsymbol{d}+\boldsymbol{z} .
$$

This final formulation represents the introduced spatio-temporal sys-

\footnotetext{
${ }^{2}$ However, it should be stressed that the proposed scheme is general and applies to any non-linear model.
} 


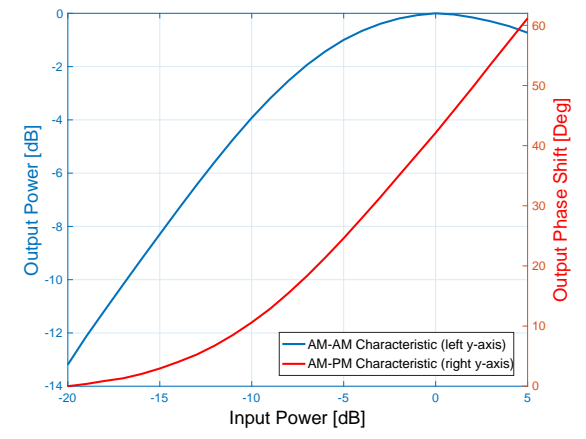

Fig. 2: Normalized AM-AM and AM-PM characteristics of the nonlinearized TWTA of [18].

tem model in a very simple way, formally similar to the spatial model used in the previous SLP literature. The matrix $\boldsymbol{G}=\boldsymbol{H} \otimes \boldsymbol{A}^{T} \in$ $\mathbb{C}^{K S \times N S}$ is an equivalent representation of the channel matrix in this novel spatio-temporal model, therefore it will be referred to as spatio-temporal channel matrix.

\section{SPATIO-TEMPORAL PEAK-TO-AVERAGE POWER RATIO MINIMIZATION}

In this section, we propose a SLP scheme relying on the introduced spatio-temporal model. The scheme performs the minimization of the PAPR of the transmitted waveforms both in the spatial and in the temporal dimensions, under QoS constraints. Moreover, in line with the previous works on SLP [7], it targets a constructive interference effect at each receiver and for each symbol slot. Herein, the formulation is provided assuming an APSK modulation scheme for the data information ${ }^{3}$. The spatio-temporal PAPR (ST-PAPR) can be simply defined, based on the vectorized communication model of (4), as $\frac{\|\boldsymbol{x}\|_{\infty}^{2}}{\|\boldsymbol{x}\|^{2} / N}$. Taking also into account the relation between $\boldsymbol{x}$ and $\boldsymbol{d}$, the optimization problem, referred to as ST-PAPR-Min, can be formulated as a non-linear fractional program as follows:

$$
\begin{array}{rc}
\boldsymbol{d}\left(\boldsymbol{s}, \boldsymbol{H}, \boldsymbol{A}_{\mathrm{TX}}, \boldsymbol{A}_{\mathrm{RX}}, \boldsymbol{\gamma}\right)=\arg \min _{\boldsymbol{d}} \frac{\left\|\left(\boldsymbol{I}_{N} \otimes \boldsymbol{A}_{\mathrm{TX}}^{T}\right) \boldsymbol{d}\right\|_{\infty}^{2}}{\left\|\left(\boldsymbol{I}_{N} \otimes \boldsymbol{A}_{\mathrm{TX}}^{T}\right) \boldsymbol{d}\right\|^{2}} \\
\text { s.t. } \quad \mathcal{C} 1: \quad\left|\boldsymbol{g}_{j i} \boldsymbol{d}\right|^{2} \unrhd \kappa_{j i}^{2} \gamma_{j}, j=1, \ldots, K, i=1, \ldots, S, \\
\mathcal{C} 2: \quad \angle \boldsymbol{g}_{j i} \boldsymbol{d}=\angle s_{j}[i], j=1, \ldots, K, i=1, \ldots, S,
\end{array}
$$

where $\boldsymbol{g}_{j i}$ denotes the spatio-temporal channel related to the $j$-th user for the $i$-th symbol slot, thus it is the $[(j-1) S+i]$-th row of $\boldsymbol{G}$, and $s_{j}[i]$ is the $i$-th element of $\boldsymbol{s}_{j}$. Further, $\gamma_{j}$ is the target signalto-interference-plus-noise ratio (SINR) that should be granted for the $j$-th user, $\gamma=\left[\gamma_{1} \ldots \gamma_{K}\right]^{T} \in \mathbb{C}^{K \times 1}$ stacks the target SINR for all the users, and $\kappa_{j i}=\left|s_{j}[i]\right| / \sqrt{\mathbb{E}_{j, i}\left[\left|s_{j}[i]\right|^{2}\right]}$ is a magnitude scaling factor for the symbol $s_{j}[i]$, which allows to account the different amplitudes of the symbols in the multi-level constellation (see also [7]). The notation $\unrhd$ represents a generalized inequality, to be read as $\geq$ or $=$ depending whether the constraint is referred to a boundary symbol or to an inner symbol of the constellation, respectively. The set of constraints $\mathcal{C} 1$ represents a QoS constraint for each user, while the set of constraints $\mathcal{C} 2$ represents the constructive interference condition, guaranteeing that each user receives the desired data symbol

${ }^{3}$ However, it can be easily extended to QAM modulations, as in [7]. with the correct phase. The optimization problem (5) is formally similar to the spatial PAPR minimization problem addressed in [12]. Thus, it can be solved following the same strategy. In particular, as a first step we can rewrite the problem by reformulating the constraints, as follows ${ }^{4}$ :

$$
\begin{aligned}
\boldsymbol{d}= & \arg \min _{\boldsymbol{d}} \frac{\|\boldsymbol{x}\|_{\infty}^{2}}{\|\boldsymbol{x}\|^{2}} \\
\text { s.t. } \quad \mathcal{C} 1: \quad & \operatorname{Re}\left(s_{j}[i]\right) \frac{\boldsymbol{g}_{j i} \boldsymbol{d}+\boldsymbol{d}^{\dagger} \boldsymbol{g}_{j i}^{\dagger}}{2} \unrhd \sqrt{\gamma_{j}} \operatorname{Re}^{2}\left(s_{j}[i]\right), \\
& j=1, \ldots, K, i=1, \ldots, S, \\
\mathcal{C} 2: \quad & \operatorname{Im}\left(s_{j}[i]\right) \frac{\boldsymbol{g}_{j i} \boldsymbol{d}-\boldsymbol{d}^{\dagger} \boldsymbol{g}_{j i}^{\dagger}}{2 \iota} \unrhd \sqrt{\gamma_{j}} \operatorname{Im}^{2}\left(s_{j}[i]\right), \\
& j=1, \ldots, K, i=1, \ldots, S, \\
\mathcal{C} 3: \quad & \left(\iota t_{j i}-1\right) \boldsymbol{g}_{j i} \boldsymbol{d}+\left(\iota t_{j i}+1\right) \boldsymbol{d}^{\dagger} \boldsymbol{g}_{j i}^{\dagger}=0, \\
& j=1, \ldots, K, i=1, \ldots, S,
\end{aligned}
$$

where $t_{j i}=\tan \left(\angle s_{j}[i]\right)$, and $\boldsymbol{x}$ has been used in place of $\left(\boldsymbol{I}_{N} \otimes \boldsymbol{A}_{\mathrm{TX}}^{T}\right) \boldsymbol{d}$ to simplify the notation.

The formulation in (6) is still challenging because of the nonlinear fractional objective function. The problem can be solved based on the method followed in $[12,13]$, by applying in a joint fashion parametric programming [19] and successive convex approximation (SCA) [20]. Accordingly, by first approximating the function $\|\boldsymbol{x}\|^{2}$ with an affine function around a generic point $\boldsymbol{z} \in \mathbb{C}^{N \xi S \times 1}$, and by then applying parametric programming using an auxiliary variable $\lambda$, we can define the optimization function $F(\lambda, \boldsymbol{z})=\min _{\boldsymbol{d} \in \mathcal{S}}\left\{\|\boldsymbol{x}\|_{\infty}^{2}-\lambda\left(2 \operatorname{Re}\left(\boldsymbol{z}^{\dagger} \boldsymbol{x}\right)-\boldsymbol{z}^{\dagger} \boldsymbol{z}\right)\right\}$, where $\mathcal{S}$ represents the sets of constraints $\mathcal{C}_{1}, \mathcal{C}_{2}, \mathcal{C}_{3}$. This yields the algorithm summarized in Table 1 , where $\lambda$ and $z$ are iteratively updated until convergence. The reader is referred to $[12,13]$ for a more detailed derivation of the algorithm.

The parametric problem (7) is convex and can be solved using the standard convex optimization tools [21]. Both the SCA approach [20] and the parametric programming [19] have been proven convergent. Moreover, a numerical analysis has evidenced a fast convergence of the proposed joint algorithm [13].

\section{NUMERICAL RESULTS}

In this section we present numerical results to assess the performance of the proposed ST-PAPR-Min scheme over non-linear channels. The presented results are obtained considering a scenario with $N=5$ antennas and $K=3$ users, a 16-APSK modulation scheme for the data information, and a block length of $S=100$ symbols, averaging over different realization of the data $\boldsymbol{S}$. The pulse shaping operation is performed using square root raised cosine (SRRC) pulses with a roll-off factor of 0.25 , while the oversampling factor $\xi$ is set to 4 . The target SINR, assumed the same for all the users, is set to $12 \mathrm{~dB}$, while for the spatial channel coefficients we have considered for simplicity a fixed realization ${ }^{5}$ as in (8). We consider as benchmarks the spatial PAPR minimization (SPAPR-Min) scheme of [12], which minimizes the PAPR only in the spatial dimension, and the peak power minimization (PPM) scheme of [10], which aims at minimizing the power peaks again only in the spatial dimension.

\footnotetext{
${ }^{4}$ The reader is referred to [12] for a more detailed derivation.

${ }^{5}$ However, the main conclusions of this work still apply if more general channel conditions are considered.
} 


$$
\boldsymbol{H}=\left[\begin{array}{ccccc}
0.2174-0.0018 \iota & -1.3545-0.6424 \iota & 0.5872-0.0178 \iota & -0.3634-0.3117 \iota & -0.3564+0.6348 \iota \\
-0.6673-0.6922 \iota & 0.3418-0.3554 \iota & -0.7173+1.1137 \iota & 0.8172+0.1664 \iota & 0.5835+0.3848 \iota \\
0.0059-1.2106 \iota & 0.7984-0.1389 \iota & 0.3298-0.3151 \iota & 0.3268+0.7113 \iota & 0.1923-1.5710 \iota
\end{array}\right]
$$

Table 1: Proposed Iterative Algorithm

1. Initialization: Set $\epsilon, k=0, \lambda=0$, which results in solving $\min _{\boldsymbol{d} \in \mathcal{S}}\left\{\|\boldsymbol{x}\|_{\infty}^{2}\right\}$.

2. Evaluate $\lambda_{0}=\frac{\|\boldsymbol{x}\|_{\infty}^{2}}{\|\boldsymbol{x}\|^{2}}, \boldsymbol{z}_{0}=\boldsymbol{x}$.

3. Solve the following optimization:

$$
\begin{aligned}
& \boldsymbol{d}=\arg \min _{\boldsymbol{d}}\|\boldsymbol{x}\|_{\infty}^{2}-\lambda_{k}\left(2 \operatorname{Re}\left(\boldsymbol{z}_{k}^{\dagger} \boldsymbol{x}\right)-\boldsymbol{z}_{k}^{\dagger} \boldsymbol{z}_{k}\right) \\
& \text { s.t. } \quad \mathcal{C} 1, \mathcal{C} 2, \mathcal{C} 3 .
\end{aligned}
$$

4. Evaluate $\left|F\left(\lambda_{k}, \boldsymbol{z}_{k}\right)\right|$ and $\left\|\boldsymbol{x}-\boldsymbol{z}_{k}\right\|$; if $\left|F\left(\lambda_{k}, \boldsymbol{z}_{k}\right)\right| \geq \epsilon$ or $\left\|\boldsymbol{x}-\boldsymbol{z}_{k}\right\| \geq \epsilon$ go to step 5 .

5. Set $\lambda_{k+1}=\frac{\|\boldsymbol{x}\|_{\infty}^{2}}{\|\boldsymbol{x}\|^{2}}, \boldsymbol{z}_{k+1}=\boldsymbol{x}, k=k+1$, go to step 3 .

Table 2: PAPR and transmit power for the different schemes.

\begin{tabular}{cccc}
\hline & ST-PAPR-Min & SPAPR-Min & PPM \\
\hline ST-PAPR [dB] & 1.05 & 7.6 & 6.5 \\
Temp. PAPR [dB] & 1.09 & 7.3 & 5.8 \\
Per-ant. Power [dBW] & 14.4 & 9.4 & 6 \\
\hline
\end{tabular}

First of all, we compare in Table 2 the spatio-temporal PAPR, in $\mathrm{dB}$, achieved by the proposed scheme and the benchmarks, together with the average per-antenna transmit power in dBW. Remarkably, the proposed scheme manages to reduce the ST-PAPR of 6.55 and $5.45 \mathrm{~dB}$ compared to the two considered benchmarks, respectively, at the expense of a higher transmit power. The table also shows the attained temporal PAPR averaged between the antennas, in order to highlight that impressive gains are obtained in the temporal dimension for each RF chain. The improved power dynamic obtained with the proposed scheme in the temporal dimension is clearly visible in Fig. 3, where the complementary cumulative distribution function (CCDF) of the instantaneous power transmitted by one antenna $\left(\left|x_{1}[l]\right|^{2}\right.$, considering the antenna indexed by 1 , with $l$ being the sample index) is drawn. Notably, the ST-PAPR-Min approach shows a much lower power variation in time.

Finally, to validate the performance of the proposed scheme with respect to non-linearities, we show in Fig. 4 the symbol error rate (SER) attained by the proposed approach and by the benchmarks over a channel corrupted by the non-linear model of Fig. 2. The SER is shown as a function of the input back-off (IBO), in $\mathrm{dB}$, applied to the signal feeding the non-linear HPAs. This result shows how the improved PAPR properties achieved by the proposed scheme result in a remarkable SER reduction when non-linearities are considered.

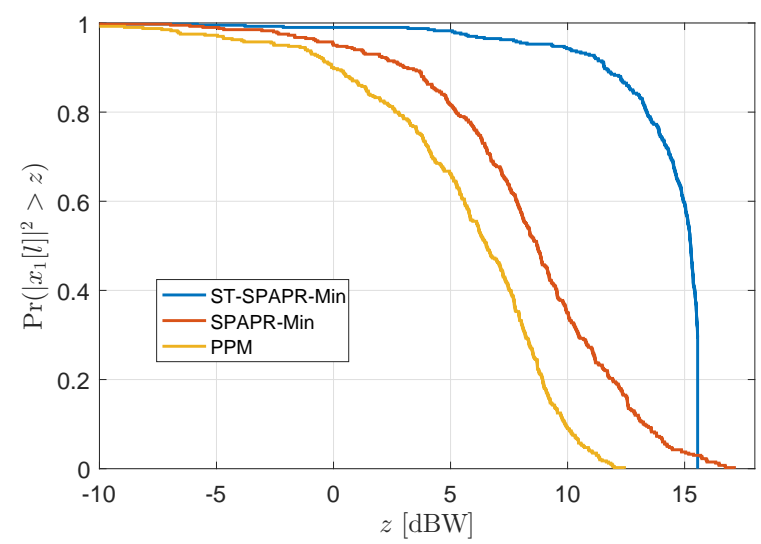

Fig. 3: CCDF of the instantaneous transmit power for a single antenna.

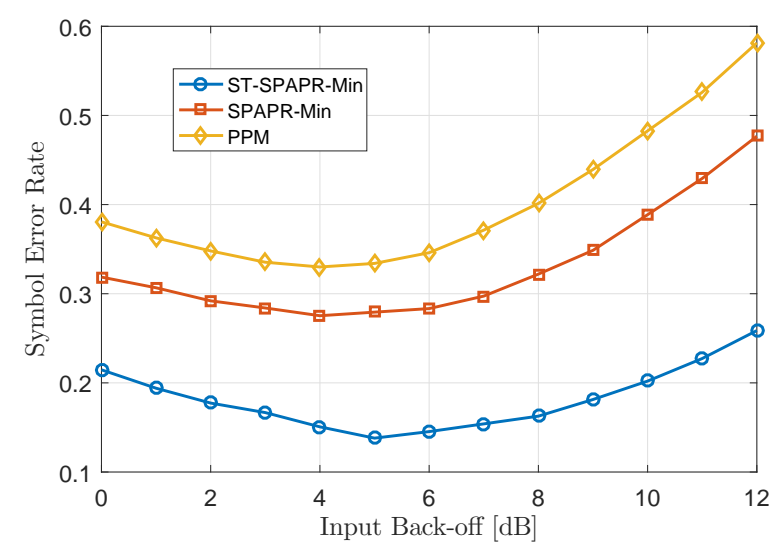

Fig. 4: Achieved SER versus IBO, in $\mathrm{dB}$, using the non-linear model of Fig. 2.

\section{CONCLUSIONS}

In this work, a novel SLP strategy has been proposed, which allows to optimize the power dynamic of the waveforms both in the spatial and in the temporal dimensions, thus improving the robustness of the signals to non-linear effects with respect to the state of the art in SLP. In particular, the introduced spatio-temporal precoding model is utilized to minimize the spatio-temporal PAPR guaranteeing some specific Quality-of-Service targets, while at the same time exploiting the constructive interference effect typical of SLP. Numerical results have been presented, in terms of PAPR, power distribution, and symbol error rate over a non-linear channel, showing remarkable gains with respect to the SLP approaches available in the literature for nonlinear channels. 


\section{REFERENCES}

[1] M. Bengtsson and B. Ottersten, "Optimal and suboptimal transmit beamforming," in Handbook of Antennas in Wireless Communications. CRC Press, 2001.

[2] M. Schubert and H. Boche, "Solution of the multiuser downlink beamforming problem with individual SINR constraints," IEEE Transactions on Vehicular Technology, vol. 53, no. 1, pp. 18-28, Jan 2004.

[3] W. Yu and T. Lan, "Transmitter optimization for the multiantenna downlink with per-antenna power constraints," IEEE Transactions on Signal Processing, vol. 55, no. 6, pp. 26462660, June 2007.

[4] C. Masouros and E. Alsusa, "Dynamic linear precoding for the exploitation of known interference in MIMO broadcast systems," IEEE Transactions on Wireless Communications, vol. 8, no. 3, pp. 1396-1404, March 2009.

[5] C. Masouros, "Correlation rotation linear precoding for MIMO broadcast communications," IEEE Transactions on Signal Processing, vol. 59, no. 1, pp. 252-262, Jan. 2011.

[6] M. Alodeh, S. Chatzinotas, and B. Ottersten, "Constructive multiuser interference in symbol level precoding for the MISO downlink channel," IEEE Transactions on Signal Processing, vol. 63, no. 9, pp. 2239-2252, May 2015.

[7] M. Alodeh, S. Chatzinotas, and B. Ottersten, "Symbol-level multiuser MISO precoding for multi-level adaptive modulation," IEEE Transactions on Wireless Communications, vol. 16, no. 8, pp. 5511-5524, Aug 2017.

[8] M. Alodeh, S. Chatzinotas, and B. Ottersten, "Energy-efficient symbol-level precoding in multiuser MISO based on relaxed detection region," IEEE Transactions on Wireless Communications, vol. 15, no. 5, pp. 3755-3767, May 2016.

[9] C. Masouros and Gan Zheng, "Exploiting known interference as green signal power for downlink beamforming optimization," IEEE Transactions on Signal Processing, vol. 63, no. 14, pp. 3628-3640, July 2015.

[10] D. Spano, M. Alodeh, S. Chatzinotas, and B. Ottersten, "Perantenna power minimization in symbol-level precoding," in 2016 IEEE Global Communications Conference (GLOBECOM), Dec 2016, pp. 1-6.

[11] D. Spano, S. Chatzinotas, J. Krause, and B. Ottersten, "Symbol-level precoding with per-antenna power constraints for the multi-beam satellite downlink," in 2016 8th Advanced Satellite Multimedia Systems Conference and the 14th Signal Processing for Space Communications Workshop (ASMS/SPSC), Sept 2016, pp. 1-8.

[12] D. Spano, M. Alodeh, S. Chatzinotas, J. Krause, and B. Ottersten, "Spatial PAPR reduction in symbol-level precoding for the multi-beam satellite downlink," in 2017 IEEE 18th International Workshop on Signal Processing Advances in Wireless Communications (SPAWC), July 2017, available at http://hdl.handle.net/10993/31224.

[13] D. Spano, M. Alodeh, S. Chatzinotas, and B. Ottersten, "Symbol-level precoding for the nonlinear multiuser MISO downlink channel," IEEE Transactions on Signal Processing, vol. 66, no. 5, pp. 1331-1345, March 2018.
[14] M. Alodeh, D. Spano, A. Kalantari, C. Tsinos, D. Christopoulos, S. Chatzinotas, and B. Ottersten, "Symbol-level and multicast precoding for multiuser multiantenna downlink: A stateof-the-art, classification and challenges," IEEE Communications Surveys \& Tutorials, 2017, submitted.

[15] G. Auer, V. Giannini, C. Desset, I. Gódor, P. Skillermark, M. Olsson, M. A. Imran, D. Sabella, M. J. Gonzalez, O. Blume, and A. Fehske, "How much energy is needed to run a wireless network?," IEEE Wireless Communications, vol. 18, no. 5, pp. 40-49, October 2011.

[16] C. Studer and E. G. Larsson, "PAR-aware large-scale multiuser MIMO-OFDM downlink," IEEE Journal on Selected Areas in Communications, vol. 31, no. 2, pp. 303-313, February 2013.

[17] M. Alodeh, D. Spano, S. Chatzinotas, and B. Ottersten, "Faster-than-Nyquist spatiotemporal symbol-level precoding in the downlink of multiuser MISO channels," in 2017 IEEE International Conference on Acoustics, Speech and Signal Processing (ICASSP), March 2017.

[18] ETSI EN 302 307-1, "Digital video broadcasting (DVB); second generation framing structure, channel coding and modulation systems for broadcasting, interactive services, news gathering and other broadband satellite applications; part 1: DVBS2," .

[19] W. Dinkelbach, "On nonlinear fractional programming," Management Science, vol. 13, no. 7, pp. 492-498, March 1967.

[20] A. Beck, A. Ben-Tal, and L. Tetruashvili, "A sequential parametric convex approximation method with applications to nonconvex truss topology design problems," Journal of Global Optimization, vol. 47, no. 01, pp. 29-51, 2010.

[21] S. Boyd and L. Vandenberghe, Convex optimization, Cambridge Univ. Press, 2004. 